\title{
Discrepancy between the initial assessment of injury severity and post hoc determination of injury severity in patients with apparently mild traumatic brain injury: a retrospective multicenter cohort analysis
}

\author{
S. M. Bossers ${ }^{1}$ - K. M. Pol ${ }^{2}$ E. P. A. Oude Ophuis ${ }^{1}$ B. Jacobs ${ }^{2}$ - M. C. Visser ${ }^{3}$. \\ S. A. Loer ${ }^{1} \cdot$ C. Boer $^{1} \cdot$ J. van der Naalt ${ }^{2} \cdot$ P. Schober $^{1}$ (I)
}

Received: 22 May 2017 / Accepted: 9 October 2017 / Published online: 14 October 2017

(C) The Author(s) 2017. This article is an open access publication

\begin{abstract}
Purpose Traumatic brain injury (TBI) is a major cause of trauma-related visits to emergency departments (ED). Determination of monitoring requirements of patients with apparently mild TBI is challenging. Patients may turn out to be more severely injured than initially assumed, and failure to identify these patients constitutes a serious threat to patient safety. We, therefore, aimed to identify clinical risk factors for more severe injuries in patients with apparently mild TBI.

Methods In a retrospective cohort analysis performed at two level I trauma centers, 808 patients aged $\geq 16$ presenting to the ED with head trauma and a Glasgow Coma Scale (GCS) score 13-15 who received a head CT scan were studied. Discrepancies between the initial TBI severity as determined by GCS and severity as determined post hoc by the Head Abbreviated Injury Score were assessed. Multiple logistic regression was used to identify risk factors of such discrepancies.

Results 104 (12.9\%) patients were more severely injured than initially classified. A GCS $<15$ at presentation (GCS 13: OR 6.2, [95\% CI 3.8-9.9]; GCS 14: OR 2.7, [2.0-3.7]), an $\mathrm{SpO}_{2}<90 \%$ (OR 5.4, [1.2-23.4]), loss of consciousness (OR 2.3, [1.5-3.5]), absence of equal and reactive pupils
\end{abstract}

P. Schober

p.schober@vumc.nl

1 Department of Anesthesiology, VU University Amsterdam Medical Center, De Boelelaan 1117, 1081 HV Amsterdam, The Netherlands

2 University of Groningen, University Medical Center Groningen, Department of Neurology, Hanzeplein 1, 9713 GZ Groningen, The Netherlands

3 Department of Neurology, VU University Medical Center, De Boelelaan 1117, 1081 HV Amsterdam, The Netherlands
(OR 2.1, [1.6-2.7]), transport by ambulance (OR 2.0, [1.7$2.4]$ ), and use of anticoagulant drugs (OR 1.2, [1.1-1.3]) were independent risk factors of more severe injury.

Conclusions Six risk factors of more severe injury in patients presenting with apparently mild TBI were identified. Patients with any of these factors should be thoroughly monitored for signs of neurologic deterioration.

Keywords Mild traumatic brain injury - Emergency medical service $\cdot$ Hospital $\cdot$ Decision support techniques · Prognosis $\cdot$ Risk factors

\section{Introduction}

Traumatic brain injury (TBI) is one of the most common injuries in the western world [1], and represents an important cause of morbidity and mortality, especially in the first decades of life [2]. TBI severity is commonly estimated using the Glasgow Coma Scale (GCS) and classified as either mild (GCS score 13-15), moderate (GCS score 9-12), or severe (GCS score 3-8) [3-5]. Patients with moderate or severe TBI, who present with impaired consciousness and often have obvious external injuries, are usually easily identified as requiring prompt attention and intensive follow-up. In contrast, determination of monitoring requirements of patients with an initial presentation of mild TBI can be more challenging. Healthcare providers need to determine which patients require additional diagnostics and monitoring, and who can be discharged safely. Patients with apparently minor injuries at the first presentation may turn out to be more severely injured than initially assumed and/or assessed [6]. Failure to identify such patients during the initial assessment can result in under-treatment and constitutes a serious threat to patient safety. Additional insights in the relationship 
between clinical parameters that are readily available to the clinician at the first presentation and injury severity may be useful to identify patients at risk and to determine monitoring requirements. We, therefore, aimed to identify clinical risk factors for a discrepancy between the initial TBI severity as determined by GCS at the initial presentation and actual injury severity as determined post hoc in patients with apparently mild TBI.

\section{Methods}

\section{Study design}

The study is a retrospective cohort analysis performed at two level I trauma centers in the Netherlands, the VU University Medical Center Amsterdam (VUmc) and the University Medical Center Groningen (UMCG). The Institutional Review Board of the VUmc determined that formal approval of the study was not required and waived the need of informed consent.

\section{Patient population}

All consecutive patients with a head trauma and who received a head CT scan between January and July 2011 in the VUmc and between January and December 2011 in the UMCG were screened for eligibility. Patients $\geq 16$ years were included if they presented with mild TBI, defined by a head trauma with a GCS score of 13-15 at the initial presentation, and if the Head Abbreviated Injury Score (H-AIS) was available. Patients presenting to the emergency department more than $24 \mathrm{~h}$ after the trauma and patients who were directly transported to another hospital after the initial assessment were not included.

\section{Definition of discrepancy between injury severity at the initial presentation and actual injury severity}

In line with common practice, we defined TBI severity at the initial assessment based on the first documented GCS score in the emergency room as described above. The Head Abbreviated Injury Score (H-AIS, version 1995, update 1998) was used as a second marker for injury severity [7]. This score is assigned in retrospect when all information is available, and is based on clinical signs and symptoms as well as pathologic-anatomical and radiologic findings [8]. Therefore, this scale cannot be used for the initial assessment, but it is useful for a post hoc determination of the actual severity. The H-AIS score ranges from 1 to 6 (minor injury to unsurvivable injury), and based on corresponding mortality rates of H-AIS and GCS, H-AIS 1-2 corresponds to mild TBI [9]. Discrepancies between the initial and post hoc classification of severity were assessed, and H-AIS scores $>2$ suggest that the patient's head injury was actually more severe than assumed during the initial assessment. The presence or absence of a discrepancy between the initial assessment of injury severity and post hoc determination of injury severity was coded as a binomial variable, which was subsequently used as dependent variable in the statistical analyses, as described in detail below.

\section{Data collection}

Data were extracted using a standardized data collection protocol from digital and paper medical records, including demographic data, injury characteristics, mode of transport to hospital (transport by ambulance vs. self-referral by private or public transport), details of physical examination, vital parameters, laboratory and radiology examination results, as well as interventions, treatments, and measures of outcome at discharge. The cerebral CT scans were scored using the Marshall classification and the Rotterdam score $[10,11]$.

\section{Statistical analysis}

Data were analyzed with STATA 13.1 (StataCorp, College Station, TX). Continuous data (e.g., age) showed significant deviations from the normal distribution. Therefore, continuous data, as well as ordinal data (e.g., GCS score), are shown as median with interquartile range; and nominal data (e.g., gender) are presented as percentages. Mann-Whitney $\mathrm{U}$ tests and Chi-square tests were used to test for differences in various parameters between patients who show and do not show a discrepancy between the initial injury severity and the injury severity as determined by the H-AIS score.

For multivariable analyses, multiple imputation was used to minimize bias due to missing data. A total of 100 data sets were imputed in which missing values were iteratively replaced using chained equations [12]. Subsequently, logistic regression, adjusted for clustering by hospital to account for the multicenter study design, was applied to the imputed data sets to address the relationship between predictor variables and discrepancies in injury severity while controlling for the effects of the other independent variables. Regression parameters were pooled across the imputed data sets to obtain an overall estimate, and Wald-test $p$ values were used to address statistical significance.

For the model development, we used a modification of the stepwise variable selection approach described by Hosmer et al. [13]: in a first step, a full model including 16 potentially relevant clinical predictor variables that are readily assessable at presentation in the emergency department was selected. To obtain a parsimonious model, stepwise backward elimination of variables with a $p$ value $>0.05$ was 
performed, until all variables in the model showed a significant $(p<0.05)$ relationship with discrepancy in injury severity. Subsequently, all previously eliminated variables were re-entered one-by-one and were retained in the model if the $p$ value provided evidence for an association with the outcome variable. This step was repeated until a final model (model A) was obtained in which all independent variables were significantly associated with the outcome variable. Goodness of fit was assessed using Hosmer-Lemeshow tests and Pearson's chi-square goodness of fit tests.

Another logistic regression model (model B) was used to determine the independent effects of the clinical predictors from model A, after adjusting for trauma-related pathology on the cerebral CT scan.

\section{Results}

A total of 808 patients matched the inclusion criteria. Of these, 498 patients were included in the VUmc and 310 in the UMCG. One hundred and four patients $(12.9 \%, 95 \%$ CI 10.6-15.2\%) showed a discrepancy between the initial severity based on the GCS at the first assessment and the actual severity as determined post hoc by the H-AIS score. Most of these patients had signs of trauma-related intracranial pathology on the initial CT scan; however, $13.4 \%$ of these patients had a normal scan. Table 1 summarizes crude demographic data as well as injury and outcome characteristics of patients with and without a discrepancy between the initial injury severity and the actual injury severity.

Table 2 shows the bivariate relationship between clinical predictor variables and discrepancies in initially assumed vs. actual injury severity after multiple imputation and accounting for clustering of the observations in two different trauma centers. Male gender, temporary postinjury loss of consciousness (LOC), amnesia, absence of pupils that are equal and reactive to light (PEARL), transport to hospital by emergency medical services (EMS), presence of hypoxia $\left(\mathrm{SpO}_{2}<90 \%\right)$, as well as any GCS lower than 15 were associated with discrepancies between assumed and actual injury severity.

In the multivariable analysis (model A), an admission GCS score of 13 and hypoxia markedly increased the odds of being more severely injured than initially assessed (OR 6.2, 95\% CI 3.8-9.9, and OR 5.4, 95\% CI 1.2-23.4, respectively). LOC, absence of PEARL, transport to hospital by EMS, and a GCS score of 14 were associated with an approximately two to threefold increase in the odds of being more severely injured. Use of anticoagulants increased the odds by approximately $20 \%$ (Table 3 ).

After adjusting for the presence of trauma-related pathology on the cerebral CT scan (model B), LOC, absence of equal and reactive pupils, transport to hospital by EMS, and hypoxia remained significantly associated with the odds of being more severely injured than initially classified (Table 3).

\section{Discussion}

We reviewed 808 patients with a clinical diagnosis of mild TBI admitted to two level I trauma centers in the Netherlands. $13 \%$ of these patients showed a discrepancy between the initial assessment of TBI severity based on the GCS, and the actual TBI severity as determined post hoc when all relevant information was available. A higher rate of hospitalization and longer hospital stay, a higher rate of admission to the intensive care unit, the increased need for neurosurgical interventions, and lower Glasgow Outcome Scale scores among patients with such a discrepancy underline that these patients are at risk for adverse outcomes and should be identified as early as possible.

Our data suggest that a GCS score $<15$, temporary postinjury LOC, absence of PEARL, hypoxia, use of anticoagulants, or admission by EMS should alarm healthcare providers that the patient may be more severely injured than initially assessed. Such clinical variables are particularly important, because patients with an apparently mild injury may not receive the highest priority in busy emergency departments, and diagnostic tests that may reveal more serious injuries can be delayed for several hours, or may not be performed at all. In our patient population, approximately $70 \%$ of patients with mild TBI received a cerebral CT scan within $2 \mathrm{~h}$ after admission, and $5 \%$ of patients waited longer than $4 \mathrm{~h}$ until being scanned.

To our knowledge, studies specifically addressing clinical predictors of a discrepancy between initially classified TBI severity and actual injury severity in patients with apparently mild TBI are lacking. The previous studies, such as the 'International Mission on Prognosis and Analysis of Clinical Trials' (IMPACT) and 'Corticoid Randomisation After Significant Head injury' (CRASH) trials, have investigated the relationship between clinical variables and adverse outcomes in patients with predominantly moderate and/or severe TBI $[14,15]$. However, outcomes as 'death' or 'severe neurologic disability' are uncommon in patients who present with an initial GCS between 13 and 15, and these studies provide only limited guidance to identify patients with apparently mild TBI who may actually be more severely injured.

Other studies have addressed the question which patients with mild TBI require a cerebral CT scan. The two most widely used scores are the Canadian CT Head Rule and the New Orleans Criteria, which have a very high sensitivity for detecting major intracranial injuries [16, 17]. Clearly, patients with apparently mild TBI who turn out to have relevant intracranial injuries are more severely injured than 
Table 1 Crude demographic data as well as injury and outcome characteristics of patients with and without a discrepancy between the initial assessment of injury severity and actual injury severity

\begin{tabular}{|c|c|c|c|c|}
\hline & No discrepancy & Discrepancy & $p$ value & $\%$ missing \\
\hline$N$ & 704 & 104 & & 0 \\
\hline \multicolumn{5}{|l|}{ Demographic characteristics } \\
\hline Age (median years) & $46(26-68)$ & $47(29-65)$ & 0.865 & 0 \\
\hline Gender (male\%/female $\%$ ) & $57 / 43$ & $73 / 27$ & 0.002 & 0 \\
\hline \multicolumn{5}{|l|}{ Anamnestic characteristics } \\
\hline Prevalence of anticoagulant use (\%) & 24.3 & 24.3 & 0.998 & 1.1 \\
\hline Loss of consciousness (\%) & 41.9 & 71.1 & $<0.001$ & 24.1 \\
\hline Amnesia (\%) & 48.5 & 78.9 & $<0.001$ & 10.8 \\
\hline Nausea $(\%)$ & 22.0 & 24.2 & 0.634 & 5.7 \\
\hline Vomiting (\%) & 8.3 & 18.2 & 0.002 & 4.6 \\
\hline Headache $(\%)$ & 35.2 & 37.8 & 0.658 & 37.0 \\
\hline PEARL $(\%)$ & 96.4 & 91.4 & 0.018 & 1.5 \\
\hline Alcohol intoxication (\%) & 38.0 & 31.6 & 0.229 & 17.0 \\
\hline Drugs intoxication $(\%)$ & 4.2 & 6.3 & 0.348 & 17.0 \\
\hline \multicolumn{5}{|l|}{ In-hospital characteristics } \\
\hline Presented to hospital by EMS (\%) & 74.2 & 85.9 & 0.012 & 7.2 \\
\hline Admission during office hours (\%) & 27.5 & 25.0 & 0.592 & 0.7 \\
\hline GCS at ED admission (median) & $15(15-15)$ & $14(14-15)$ & $<0.001$ & 0 \\
\hline GCS $15(\%)$ & 79.3 & 49.0 & & \\
\hline GCS $14(\%)$ & 17.2 & 35.6 & & \\
\hline GCS $13(\%)$ & 3.6 & 15.4 & & \\
\hline SBP on ED admission (median $\mathrm{mmHg}$ ) & $140(122-155)$ & $130(117-150)$ & 0.089 & 15.3 \\
\hline Hypotension (SPB < 100 mmHg) (\%) & 3.4 & 1.1 & & \\
\hline Normotension $(\%)$ & 78.7 & 79.6 & & \\
\hline Hypertension (SPB > 160 mmHg) $(\%)$ & 18.0 & 19.3 & & \\
\hline DBP on ED admission (median $\mathrm{mmHg}$ ) & $80(70-90)$ & $80(70-90)$ & 0.923 & 15.3 \\
\hline Heart rate on ED admission (median $\min ^{-1}$ ) & $80(71-92)$ & $80(70-90)$ & 0.515 & 16.5 \\
\hline Bradycardia (HR < 60/min) $(\%)$ & 3.9 & 4.5 & & \\
\hline Normocardia $(\%)$ & 85.2 & 84.3 & & \\
\hline Tachycardia (HR > 100/min) $(\%)$ & 10.9 & 11.2 & & \\
\hline $\mathrm{SpO}_{2}$ on ED admission (median \%) & $99(97-100)$ & $99(97-100)$ & 0.311 & 20.9 \\
\hline Hypoxia $\left(\mathrm{SpO}_{2}<90 \%\right)(\%)$ & 0.7 & 3.5 & 0.021 & \\
\hline Hospitalized (\%) & 47.5 & 97.1 & $<0.001$ & 0.4 \\
\hline Hospitalization days (median) & $0(0-2)$ & $3(2-9)$ & $<0.001$ & 6.2 \\
\hline ICU admission (\%) & 2.5 & 10.8 & $<0.001$ & 1.5 \\
\hline Neurosurgical Intervention (\%) & 0.1 & 6.9 & $<0.001$ & 1.4 \\
\hline \multicolumn{5}{|l|}{ Characteristics of the initial cerebral CT scan } \\
\hline Time to first CT scan (median minutes) & 82 & 61 & $<0.001$ & 8.4 \\
\hline Any trauma-related pathology $(\%)$ & 7.7 & 86.6 & $<0.001$ & 4.3 \\
\hline Skull fracture $(\%)$ & 12.8 & 35.7 & $<0.001$ & 30.8 \\
\hline Rotterdam CT score (median) & $1(1-2)$ & $3(2-3)$ & $<0.001$ & 5.8 \\
\hline Marshall CT classification (\%) & & & $<0.001$ & 5.7 \\
\hline I & 96.3 & 19.8 & & \\
\hline II & 3.4 & 68.1 & & \\
\hline III & 0.2 & 5.5 & & \\
\hline IV & 0.2 & 0 & & \\
\hline VI & 0 & 6.6 & & \\
\hline Requirement for second CT scan (\%) & 1.8 & 27.0 & $<0.001$ & 3.5 \\
\hline \multicolumn{5}{|l|}{ Injury severity and Outcome } \\
\hline H-AIS (median) & $2(1-2)$ & $3(3-4)$ & $<0.001$ & 0 \\
\hline Injury Severity Score (median) & $5(3-8)$ & $16(10-25)$ & $<0.001$ & 0.4 \\
\hline
\end{tabular}


Table 1 (continued)

\begin{tabular}{lllll}
\hline & No discrepancy & Discrepancy & $p$ value & $\%$ missing \\
\hline Glasgow Outcome Score (median) & $5(5-5)$ & $5(4-5)$ & $<0.001$ & 1.5 \\
Glasgow Outcome Score $<5(\%)$ & 8.4 & 37.3 & $<0.001$ & 1.5 \\
In-hospital mortality $(\%)$ & 0.4 & 1.9 & 0.069 & 1.0 \\
\hline
\end{tabular}

Anticoagulant use refers to the use of anticoagulant or antiplatelet drugs before the injury. Alcohol intoxication was assumed based on anamnestic data or by a blood alcohol concentration $\geq 0.05 \%$. Drug Intoxication was determined based on anamnestic data or on positive drug screening results

PEARL 'Pupils equal and reactive to light', EMS Emergency Medical Services, Office hours Monday-Friday from 8 a.m. to 5 p.m., GCS Glasgow Coma Scale, ED Emergency Department, SBP systolic blood pressure, $D B P$ diastolic blood pressure, $I C U$ intensive care unit, $H$-AIS head abbreviated injury score

initially assumed. Yet, however, predictors of injuries on CT scans are not necessarily identical to clinical risk factors for the presence of injuries that are more severe than initially assumed in patients with apparently mild TBI. First, the studies in which the scores were developed limited inclusion to patients with LOC or amnesia, implying that patients without these signs are not at risk of having significant injuries. In contrast, the absence of these features does not exclude intracranial injuries $[6,18]$. We, therefore, did not restrict inclusion to patients with LOC or amnesia, and in fact, in the present study, about $8 \%$ of the patients with a discrepancy between initially assumed and actual injury severity neither had LOC nor amnesia. Second, a normal initial CT scan does not exclude a clinically important injury, including delayed hemorrhage, which may still even occur up to several weeks after the initial trauma, or nonhemorrhagic injuries, such as traumatic axonal injury [19, 20]. In fact, $13 \%$ of the patients who turned out to be more severely injured than assumed in our patient population had an unremarkable initial CT scan.

This underlines that clinical variables may be a useful supplement to identify patients at risk. Not surprisingly, a part of the risk factors that we identified, such as absence of PEARL, LOC, and hypoxia, have previously been associated with adverse outcomes after TBI [2, 6, 14, 15]. Surprisingly, preinjury use of anticoagulant drugs was not a significant risk factor in the bivariate analysis, even though several previous studies suggest worse outcome after TBI in patients using anticoagulant drugs [21-23]. However, note that the bivariate analysis is subject to confounding and must be interpreted with care. After adjustment for other risk factors in the multivariable model, preinjury use of anticoagulants was, indeed, significantly associated with an approximately $20 \%$ higher odds to be more severely injured than initially assumed. Therefore, preinjury use of anticoagulants actually does need to be considered in the assessment of patients with apparently mild TBI.

To our knowledge, transport by EMS has previously not been studied as a potential risk factor for adverse outcomes. Interestingly, EMS transport was independently associated with a two-fold increase in the odds of a discrepancy between initially assumed and actual injury severity, and this also holds true after adjusting for the initial CT scan. Patients transported by EMS were comparable to patients who were not transported by EMS with respect to age, GCS scores, incidence of LOC, incidence of hypo-/hypertension, incidence of brady-/tachycardia, incidence of hypoxia, as well as incidence of use of anticoagulant drugs. Yet, these patients have an increased risk to be more severely injured than initially assessed. A likely explanation is that the variable EMS transport represents a surrogate for other (unobserved) variables that may be difficult to quantify, but which yet indicate more severe injury. Transport by EMS suggests that patients or bystanders perceived the accident as severe enough to warrant EMS activation, and that EMS personnel perceived the accident as severe enough to transport the patient by ambulance. It is not always clear in retrospect what the subjective or objective criteria were to transport a certain patient by ambulance, while EMS were not activated for another similar patient. Whatever the reasons may have been, our data suggest that patients who are presented by EMS should be closely monitored as they may be more severely injured than assumed.

\section{Limitations}

We performed a retrospective study, with the inherent limitations of this research design. To minimize selection bias, all consecutive patients meeting the inclusion criteria were included. Nonetheless, the inclusion criteria may have led to some selection bias as we included only patients who received a CT scan. The decision to perform a CT scan was made using local protocols in the trauma centers, which are based on a guideline by the Dutch Society of Neurology [24]. According to this guideline, a CT scan is indicated for all patients with a GCS score $<15$ at presentation, such that selection bias should only not have affected patients with a GCS score of 13 or 14. Patients with a GCS score of 15 also regularly receive a CT scan (609 patients in our study had a GCS score of 15), as the guideline defines a variety of other criteria (such as trauma mechanism, clinical signs 
Table 2 Bivariate relationship between potential clinical predictor variables and discrepancies between initially assumed vs. actual injury severity, after multiple imputation and adjustment for clustering of the observations in two different trauma centers

\begin{tabular}{llr}
\hline & OR $(95 \%$ CI $)$ & $p$ value \\
\hline Clinical variables & & \\
Male gender & $2.1(1.2-3.6)$ & 0.011 \\
Age $\geq 50$ years & $1.0(0.5-2.0)$ & 0.999 \\
Age $\geq 65$ years & $0.9(0.4-2.4)$ & 0.886 \\
Age (continuous) & $1.0(1.0-1.0)$ & 0.958 \\
Use of anticoagulants & $1.0(0.7-1.4)$ & 0.947 \\
Loss of consciousness & $2.8(1.8-4.3)$ & $<0.001$ \\
Amnesia & $3.8(1.3-10.6)$ & 0.012 \\
Nausea & $1.1(0.4-2.9)$ & 0.807 \\
Vomiting & $2.4(0.9-6.5)$ & 0.085 \\
Headache & $1.2(0.6-2.2)$ & 0.614 \\
Absence of PEARL & $2.5(2.2-2.8)$ & $<0.001$ \\
Alcohol Intoxication & $0.8(0.4-1.6)$ & 0.623 \\
Drugs Intoxication & $1.5(0.7-3.3)$ & 0.286 \\
Presented to hospital by EMS & $2.1(1.4-3.0)$ & $<0.001$ \\
Admission during office hours & $0.9(0.6-1.3)$ & 0.494 \\
Systolic blood pressure & & \\
Normotension & Reference & \\
Hypotension & $0.5(0.1-3.3)$ & 0.508 \\
Hypertension & $1.0(0.4-2.4)$ & 0.954 \\
Heart rate & & \\
Normocardia & Reference & \\
Bradycardia & $1.1(0.4-2.9)$ & 0.835 \\
Tachycardia & $1.0(0.7-1.4)$ & 0.0067 \\
Hypoxia (SpO $2<90 \%)$ & $4.8(1.6-14.6)$ & \\
GCS at ED admission & & \\
15 & & \\
14 & & \\
13 & & \\
\hline
\end{tabular}

Anticoagulant use refers to the use of anticoagulant or antiplatelet drugs before the injury. Alcohol intoxication was assumed based on anamnestic information or by a blood alcohol concentration $\geq 0.05 \%$. Drug Intoxication was determined based on anamnestic information or on positive drug screening results. Normotension: systolic blood pressure $100-160 \mathrm{mmHg}$, Normocardia: hart rate $60-100$ beats per $\min$

PEARL 'Pupils equal and reactive to light', EMS Emergency Medical Services, Office hours Monday-Friday from 8 a.m. to 5 p.m., GCS Glasgow Coma Scale, ED Emergency Department

of head injury, age, vomiting, temporary loss of consciousness, amnesia, or focal neurologic symptoms) which alone or in combination are used to determine the indication for a CT scan. Nonetheless, we may have missed patients in our analysis who presented to the ED with a trivial head trauma and a GCS score of 15 .

To minimize information bias, we extracted and crosschecked the data across multiple data sources (electronic and
Table 3 multivariable logistic regression showing the relationship between several clinical predictor variables and discrepancies between the initial assessment of injury severity and actual injury severity (Model A)

\begin{tabular}{lll}
\hline & OR $(95 \%$ CI $)$ & $p$ value \\
\hline Model A & & \\
Use of anticoagulants & $1.2(1.1-1.3)$ & 0.004 \\
Loss of consciousness & $2.3(1.5-3.5)$ & $<0.001$ \\
Absence of PEARL & $2.1(1.6-2.7)$ & $<0.001$ \\
Presented to hospital by EMS & $2.0(1.7-2.4)$ & $<0.001$ \\
Hypoxia (SpO 2 <90\%) & $5.4(1.2-23.4)$ & 0.026 \\
GCS at ED admission & & \\
15 & Reference & \\
14 & $2.7(2.0-3.7)$ & $<0.001$ \\
13 & $6.2(3.8-9.9)$ & $<0.001$ \\
Model B & & \\
Use of anticoagulants & $1.1(0.9-1.3)$ & 0.399 \\
Loss of consciousness & $1.9(1.1-3.3)$ & 0.032 \\
Absence of PEARL & $2.3(1.4-3.6)$ & $<0.001$ \\
Presented to hospital by EMS & $2.3(1.4-3.6)$ & 0.001 \\
Hypoxia (SpO $2<90 \%)$ & $4.2(1.5-11.8)$ & 0.014 \\
GCS at ED admission & & \\
15 & Reference & \\
14 & $1.7(0.8-4.0)$ & 0.190 \\
13 & $2.1(0.6-7.1)$ & 0.213 \\
Pathology on cerebral CT scan & $66.2(19.1-229)$ & $<0.001$ \\
\hline
\end{tabular}

All estimates are adjusted for the effects of the other independent variables in the model, and are corrected for clustering of the observations in two different trauma centers. Model B is additionally adjusted for the presence of trauma-related pathology on the CT scan. Anticoagulant use refers to the use of anticoagulant or antiplatelet drugs before the injury

PEARL 'Pupils equal and reactive to light', EMS Emergency Medical Services, GCS Glasgow Coma Scale, ED Emergency Department

hand-written medical records, and hospital's trauma registration system) using a standardized data coding protocol. Nonetheless, we could not avoid missing data and, therefore, used multiple imputation to increase efficiency and to minimize bias which could occur in complete case analysis [25].

We compared the initial GCS scores at admission to H-AIS scores that were determined post hoc. While both scores are used to quantify injury severity, they do not measure exactly the same quantity and the comparison may at the first glance seem as comparing apples to oranges. However, we were specifically interested to assess discrepancies between the initial assessment of injury severity and the actual severity of the injury. The former is addressed by the GCS score at presentation, and the latter is characterized by the H-AIS score, which is scored by a specially trained and dedicated data manager of the trauma center when all information is available after diagnostics and treatment have been terminated. Hence, discrepancies between 
the initial GCS and H-AIS suggest that the patient was actually more severely injured than initially assessed, or that the patient deteriorated during hospital admission. Either way, these patients are at risk for under-treatment of injuries or complications.

Finally, we did not externally validate our results. Additional research is necessary to confirm whether the risk factors can also be used to identify patients at risk in hospitals that have a different case-mix.

\section{Conclusions}

Head injured patients with apparently mild TBI, as defined by an initial GCS score of 13-15 at presentation, have a considerable risk of being more severely injured than initially classified. A GCS score of less than 15, LOC, absence of reactive and equal pupils, hypoxia, use of anticoagulant medication, and admission by EMS were associated with an increased risk. Such patients should be thoroughly monitored for signs of neurologic deterioration. An admission $\mathrm{SpO}_{2}<90 \%$, absence of equal and reactive pupils, transport by ambulance, and loss of consciousness are factors which indicate an increased risk, independently of the presence or absence of trauma-related pathology on the initial cerebral CT scan.

Author contributions SB, BJ, MV, SL, CB, JvdN, and PS contributed to the conception and design of the study; MP, EOO, MV, and JvdN collected data; BJ, SL, and CB provided logistic support during data collection and monitored the study progress; SB and PS analyzed the data and drafted the first version of the manuscript; MP, EOO, BJ, $\mathrm{MV}, \mathrm{SL}, \mathrm{CB}$, and JvdN critically revised the manuscript for important intellectual content. All authors read and approved the final version of the manuscript.

\section{Compliance with ethical standards}

Conflicts of interest Sebastiaan M. Bossers, Klaas M. Pol, Eva Oude Ophuis, Bram Jacobs, Marieke C. Visser, Stephan A. Loer, Christa Boer, Joukje van der Naalt, and Patrick Schober declare that they have no conflict of interest

Statement of human rights and informed consent For this retrospective study of anonymized data, the Institutional Review Board of the VU University Medical Center determined that formal approval was not required and waived the need of informed consent.

Open Access This article is distributed under the terms of the Creative Commons Attribution 4.0 International License (http://creativecommons.org/licenses/by/4.0/), which permits unrestricted use, distribution, and reproduction in any medium, provided you give appropriate credit to the original author(s) and the source, provide a link to the Creative Commons license, and indicate if changes were made.

\section{References}

1. Smits M, Dippel DW, de Haan GG, Dekker HM, Vos PE, Kool DR, et al. External validation of the Canadian CT Head Rule and the New Orleans Criteria for CT scanning in patients with minor head injury. Jama. 2005;294(12):1519-25. doi:10.1001/ jama.294.12.1519.

2. Bossers SM, Schwarte LA, Loer SA, Twisk JW, Boer C, Schober $P$. Experience in prehospital endotracheal intubation significantly influences mortality of patients with severe traumatic brain injury: a systematic review and meta-analysis. PloS One. 2015;10(10):e0141034. doi:10.1371/journal.pone.0141034.

3. Blyth BJ, Bazarian JJ. Traumatic alterations in consciousness: traumatic brain injury. Emerg Med Clin North Am. 2010;28(3):571-94. doi:10.1016/j.emc.2010.03.003.

4. Laskowski RA, Creed JA, Raghupathi R. Pathophysiology of Mild TBI: implications for altered signaling pathways. In: Kobeissy FH, editor. Brain neurotrauma: molecular, neuropsychological, and rehabilitation aspects. Frontiers in neuroengineering. Boca Raton: CRC Press; 2015. pp. 35-42.

5. Teasdale G, Jennett B. Assessment of coma and impaired consciousness. A practical scale. The Lancet. 1974;2(7872):81-4.

6. Easter JS, Haukoos JS, Meehan WP, Novack V, Edlow JA. Will neuroimaging reveal a severe intracranial injury in this adult with minor head trauma?: the rational clinical examination systematic review. Jama. 2015;314(24):2672-81. doi:10.1001/ jama.2015.16316.

7. Gennarelli TA, Wodzin E. AIS 2005: a contemporary injury scale. Injury. 2006;37(12):1083-91. doi:10.1016/j. injury.2006.07.009.

8. Timmons SD, Bee T, Webb S, Diaz-Arrastia RR, Hesdorffer D. Using the abbreviated injury severity and Glasgow Coma Scale scores to predict 2-week mortality after traumatic brain injury. J Trauma. 2011;71(5):1172-8. doi:10.1097/TA.0b013e31822b0f4b.

9. Demetriades D, Kuncir E, Murray J, Velmahos GC, Rhee P, Chan L. Mortality prediction of head Abbreviated Injury Score and Glasgow Coma Scale: analysis of 7764 head injuries. J Am Coll Surg. 2004;199(2):216-22. doi:10.1016/j. jamcollsurg.2004.02.030.

10. Maas AI, Hukkelhoven CW, Marshall LF, Steyerberg EW. Prediction of outcome in traumatic brain injury with computed tomographic characteristics: a comparison between the computed tomographic classification and combinations of computed tomographic predictors. Neurosurgery. 2005;57(6):1173-82. (discussion-82).

11. Marshall LF, Marshall SB, Klauber MR, Van Berkum Clark M, Eisenberg H, Jane JA, et al. The diagnosis of head injury requires a classification based on computed axial tomography. J Neurotrauma. 1992;9(Suppl 1):S287-92.

12. Azur MJ, Stuart EA, Frangakis C, Leaf PJ. Multiple imputation by chained equations: what is it and how does it work? Int J Methods Psychiatr Res. 2011;20(1):40 - 9. doi:10.1002/mpr.329.

13. Hosmer DW, Lemeshow S, Sturdivant RX. Model-building strategies and methods for logistic regression. applied logistic regression. 3rd ed. Hoboken: Wiley; 2013. pp. 89-152.

14. Trial Collaborators MRC CRASH, Perel P, Arango M, Clayton T, Edwards P, Komolafe E, et al. Predicting outcome after traumatic brain injury: practical prognostic models based on large cohort of international patients. BMJ. 2008;336(7641):425-9. doi:10.1136/ bmj.39461.643438.25.

15. Murray GD, Butcher I, McHugh GS, Lu J, Mushkudiani NA, Maas AI, et al. Multivariable prognostic analysis in traumatic brain injury: results from the IMPACT study. J Neurotrauma. 2007;24(2):329-37. doi:10.1089/neu.2006.0035. 
16. Haydel MJ, Preston CA, Mills TJ, Luber S, Blaudeau E, DeBlieux $\mathrm{PM}$. Indications for computed tomography in patients with minor head injury. N Engl J Med. 2000;343(2):100-5. doi:10.1056/ NEJM200007133430204.

17. Stiell IG, Wells GA, Vandemheen K, Clement C, Lesiuk H, Laupacis A, et al. The Canadian CT head rule for patients with minor head injury. The Lancet. 2001;357(9266):1391-6.

18. Easter JS, Grossman SA, Woodruff MM, Rosen CL. When the rules do not work: head injury without loss of consciousness. J Emerg Med. 2008;35(1):77-80. doi:10.1016/j. jemermed.2008.04.002.

19. Lee B, Newberg A. Neuroimaging in traumatic brain imaging. NeuroRx J Am Soc Exp NeuroTher. 2005;2(2):372-83. doi:10.1602/neurorx.2.2.372.

20. Snoey ER, Levitt MA. Delayed diagnosis of subdural hematoma following normal computed tomography scan. Ann Emerg Med. 1994;23(5):1127-31.

21. Ohm C, Mina A, Howells G, Bair H, Bendick P. Effects of antiplatelet agents on outcomes for elderly patients with traumatic intracranial hemorrhage. J Trauma. 2005;58(3):518 - 22.
22. Pieracci FM, Eachempati SR, Shou J, Hydo LJ, Barie PS. Use of long-term anticoagulation is associated with traumatic intracranial hemorrhage and subsequent mortality in elderly patients hospitalized after falls: analysis of the New York State Administrative Database. J Trauma. 2007;63(3):519-24. doi:10.1097/ TA.0b013e31812e519b.

23. Wong DK, Lurie F, Wong LL. The effects of clopidogrel on elderly traumatic brain injured patients. J Trauma. 2008;65(6):1303-8. doi:10.1097/TA.0b013e318185e234.

24. Dutch Society of Neurology. Richtlijn opvang van patienten met licht traumatisch hoofd/hersenletsel. 2010. http://www.nvk.nl/Portals/0/richtlijnen/licht traumatisch hoofd- en hersenletsel/hoofdor-hersenletsel-licht-traumatisch.pdf. Accessed 22 May 2017.

25. Sterne JA, White IR, Carlin JB, Spratt M, Royston P, Kenward MG, et al. Multiple imputation for missing data in epidemiological and clinical research: potential and pitfalls. BMJ. 2009;338:b2393. doi:10.1136/bmj.b2393. 